\title{
Focus Magnet and Vessel Interface Issues in HYLIFE-II
}

\author{
P.A. House
}

\section{January 18, 2000}

U.S. Department of Energy

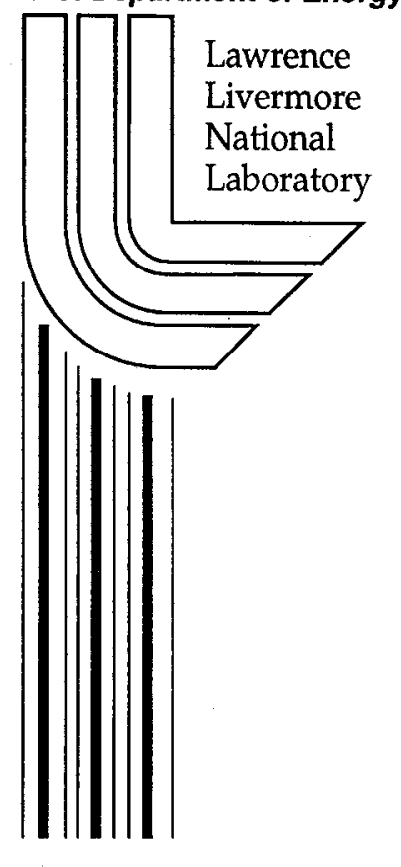




\section{DISCLAIMER}

This document was prepared as an account of work sponsored by an agency of the United States Government. Neither the United States Government nor the University of California nor any of their employees, makes any warranty, express or implied, or assumes any legal liability or responsibility for the accuracy, completeness, or usefulness of any information, apparatus, product, or process disclosed, or represents that its use would not infringe privately owned rights. Reference herein to any specific commercial product, process, or service by trade name, trademark, manufacturer, or otherwise, does not necessarily constitute or imply its endorsement, recommendation, or favoring by the United States Government or the University of California. The views and opinions of authors expressed herein do not necessarily state or reflect those of the United States Government or the University of California, and shall not be used for advertising or product endorsement purposes.

Work performed under the auspices of the U. S. Department of Energy by the University of California Lawrence Livermore National Laboratory under Contract W-7405-Eng-48.

This report has been reproduced

directly from the best available copy.

Available to DOE and DOE contractors from the

Office of Scientific and Technical Information

P.O. Box 62, Oak Ridge, TN 37831

Prices available from (423) 576-8401

http://apollo.osti.gov/bridge/

Available to the public from the

National Technical Information Service

U.S. Department of Commerce

5285 Port Royal Rd., Springfield, VA 22161

http://www.ntis.gov/

OR

Lawrence Livermore National Laboratory

Technical Information Department's Digital Library

http://www.llnl.gov/tid/Library.html 


\title{
Focus Magnet and Vessel Interface Issues in HYLIFE-II*
}

\author{
Palmer A. House \\ University of California \\ Lawrence Livermore National Laboratory \\ P.O. Box 808 \\ Livermore, California 94550 \\ January 18,2000
}

\begin{abstract}
The present Heavy Ion Driver design for HYLIFE-II calls for 96 beams from each side, or a total of 192 beams. The bcams are separated from each other, at present, by an angle of 4.25 degrees. This report shows the focus magnet locations and a magnet build that leads to the minimum angle of 4.25 degrees between beams. Beam line and first wall shielding for the oscillating flow version of HYLIFE-II is accomplished by a series of horizontal and vertical jets. Ideally the horizontal jets would not deviate from a straight line but this is not feasible due to the force of gravity. Methods of altering the beam line array pattern to accommodate the curved "horizontal" jets are addressed.
\end{abstract}

\section{Introduction}

HYLIFE-II is a design study of a $1 \mathrm{GW}_{\mathrm{e}}$ inertial fusion energy power plant (Moir, 1994). Shielding of the metal parts of the structure is accomplished by a "liquid wall" using Molten Flibe $\left(\mathrm{Li}_{2} \mathrm{BeF}_{4}\right)$. Two sets of Flibe (molten salt) jets, 90 degrees apart and vortex devices, are used for x-ray and neutron shielding of the first vessel wall (House, 1999). The heavy ion driver has evolved from 12 beams from one side (Moir, et al., 1994) to the present 96 beams per side. The difficulty in providing adequate beam line shielding increases as the space between beams decreases.

In order to investigate cross flow Flibe shielding jets in the beam entrance zones, inside the vessel, we needed to know information about the beam sizes and the angle between beams. Preliminary design information about beam dimensions (Figure 1) and focus magnet locations (Table 1) was provided by Ed Lee (Lee, 1999). The beam sizes listed in Table 1 were estimated by scaling from Figure 1. Ed Lee thought that the beam size shown in the Q5 magnet zone (Fig. 1) could be reduced.

The minimum angle between beams is dependent on the room required for close-in focus magnets. From Fig.4 one can see that the Q6 and Q5 magnets are very closely spaced compared to magnets Q1 through Q4. We estimated the size of magnet Q5 by a layout showing the beam, beam tube, various shells for cooling functions and the winding, see Figure 2. A box nearly $45 \times 45 \mathrm{~cm}$ is needed for magnet Q5. The minimum box size 
needed for Q6 is about $37 \times 37 \mathrm{~cm}$, based on the smaller beam major radius of $6.7 \mathrm{~cm}$ and a $2 \mathrm{~cm}$ thick winding. A 4.25 degree angle between beams was assigned to allow for a slightly larger space for the Q6 magnets. This angle allows for a $38.4 \times 38.4 \mathrm{~cm}$ Q6 box at the $5.18 \mathrm{~m}$ radius. The packing arrangement for the 96, Q6 magnets is shown in Fig. 3. The 4.25 degree angle between beams results in a space between Q5 magnets of $5 \mathrm{~cm}$ at $6.66 \mathrm{~m}$ from the working point.

The arrangement of focus magnets is shown in Fig. 4. Sixty of the 576 (96 x 6) focus magnets per side can be seen along with a portion of the vessel wall, vortex shielding devices, and the cross flow shielding jets.

\section{Jet Curvature}

The orthogonal shielding jets are arranged, for the oscillating central flow case, as horizontal jets near the working point, and then vertical jets, see Fig. 4. In Fig. 5 the beams are shown evenly spaced with gravity induced curved jets in between. It is apparent that the space between beams and jets varies, depending on location. Higher jet velocities would reduce the curvature but may result in less stable jets. Another factor with higher velocities is higher pumping pressure and power. Figure 6 shows beam positions adjusted to be evenly spaced between the jets. The Heavy Ion Accelerator Group suggested that the beam position adjustment could be accomplished by beam steering. It is important to have minimal and equal space between horizontal and vertical jets and the beams in order to have room for a vortex shielding device. This device shields the beam tube and a square unshielded zone around the tube. Another potential method of shifting the beam columns (a-e in Fig. 6) is to rotate slightly the focus magnet vertical arrays about the working point. The result of these magnet array rotations is shown in Fig. 7. This may cause problems in Q5 and Q6 where the intention is to share mechanical magnet forces and fields between the magnets.

Another arrangement for the orthogonal shielding jets is for them to be rotated 45 degrees, this results in each set of jets having an angle of 45 degrees to a horizontal plane. This configuration is illustrated in Fig. 8 where both sets of jets now have curvature from gravity. Once again beam steering or focus magnet position shifts will be required in order to centralize beams between the jets.

\section{Conclusions}

The purpose of this report was to document one early configuration state of the focus magnets. This allowed studies of the cross flow shielding jets and magnet radiation shielding to proceed. It is anticipated that this study will aid in modifying the design for the next iteration. 
* Work performed under the auspice of the U. S. Department of Energy by Lawrence Livermore Laboratory under Contract W-7405-ENG-48.

\section{References}

1. P. A. House, "Beam Line and First Wall Shielding in HYLIFE-II", UCRL-ID136107, October 18, 1999

2. R. W. Moir, et al. (1994), "HYLIFE-II: A Molten Salt Inertial Fusion Energy Power Plant Design-Final Report”, Fusion Technology 25, 5-25 (1994)

3. E. P. Lee, Lawrence Berkeley National Laboratory, private communication, 6/15/99. 
Table 1. Magnet locations and beam sizes

\begin{tabular}{|c|c|c|c|c|}
\hline \multicolumn{5}{|c|}{ Magnet locations (System with 96 magnets per side) } \\
\hline \multirow{2}{*}{\multicolumn{2}{|c|}{ Magnet locations: }} & & & \\
\hline & & Beam (Min.) & Beam (Max.) & Magnet Locations: \\
\hline & & & & E. Lee's Ref. Syst. \\
\hline & (m) & (cm) & (cm) & (m) \\
\hline (Focus point) & 0.0 & & & 20.6 \\
\hline Vortex & 2.4 & 1.7 & 1.7. & - \\
\hline Vortex & 2.9 & 2.1 & 2.1 & - \\
\hline Q6 & 5.18 & 3.9 & 3.9 & 15.42 \\
\hline Q6 & 6.18 & 4.0 & 6.7 & 14.42 \\
\hline Q5 & 6.66 & 3.5 & 9.3 & 13.94 \\
\hline Q5 & 7.66 & 4.1 & 10.1 & 12.94 \\
\hline Q4 & 10.02 & 5.8 & 8.6 & 10.58 \\
\hline Q4 & 11.02 & 5.6 & 9.1 & 9.58 \\
\hline Q3 & 13.38 & 5.6 & 9.1 & 7.22 \\
\hline Q3 & 14.38 & 5.8 & 8.8 & 6.22 \\
\hline Q2 & 16.74 & 5.8 & 8.9 & 3.86 \\
\hline $\mathrm{Q} 2$ & 17.74 & 5.6 & 9.1 & 2.86 \\
\hline Q1 & 20.1 & 5.6 & 8.9 & 0.5 \\
\hline Q1 & 20.6 & 5.4 & 9.4 & 0.0 \\
\hline
\end{tabular}




\section{Heavy ion beam sizes in the focus magnet zone}

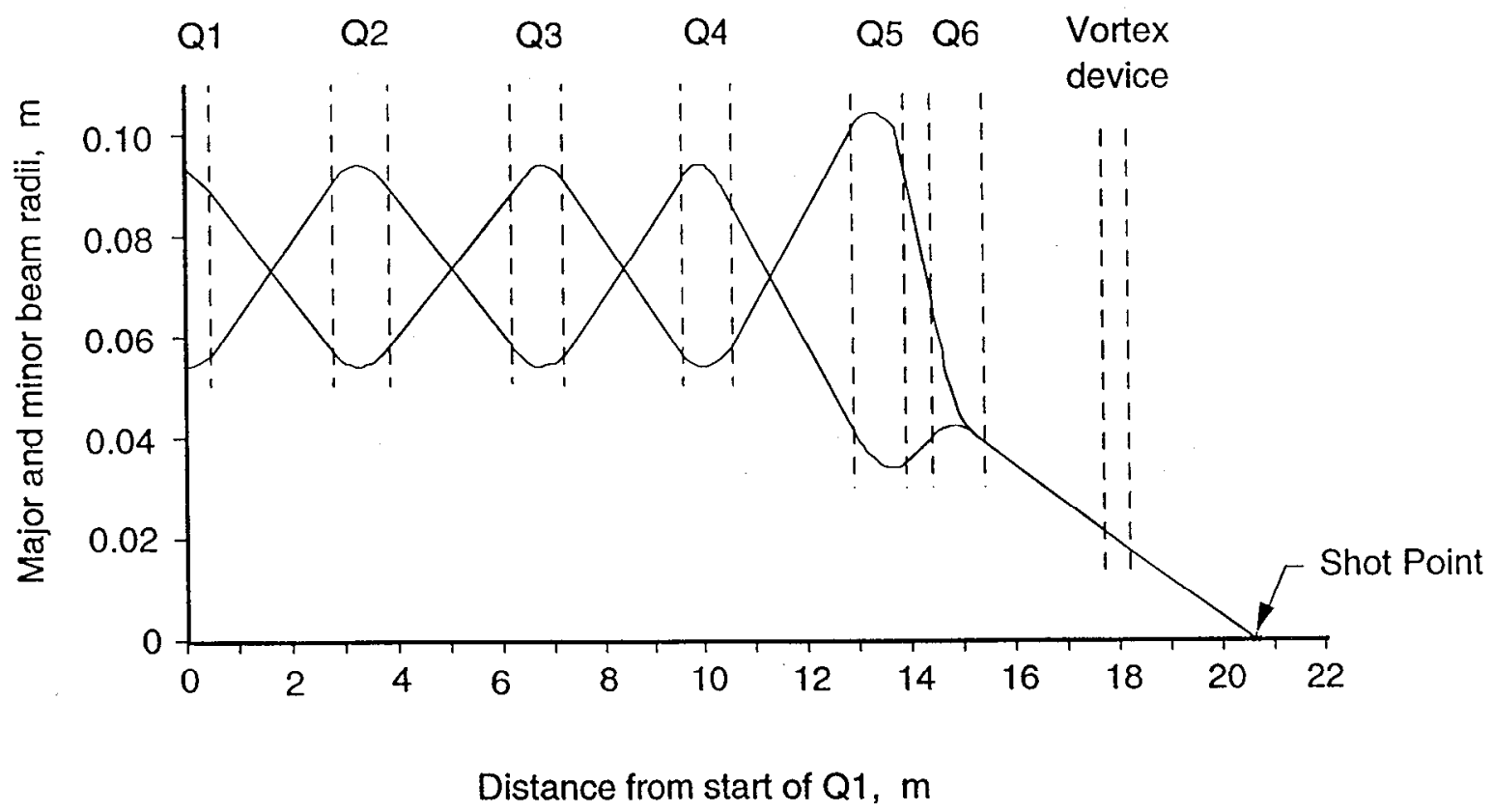

Figure 1. Beam size vs location (approximate) 


\section{Quadrupole Magnet Build (Q5, @ 6.66 m From Detonation Point)}

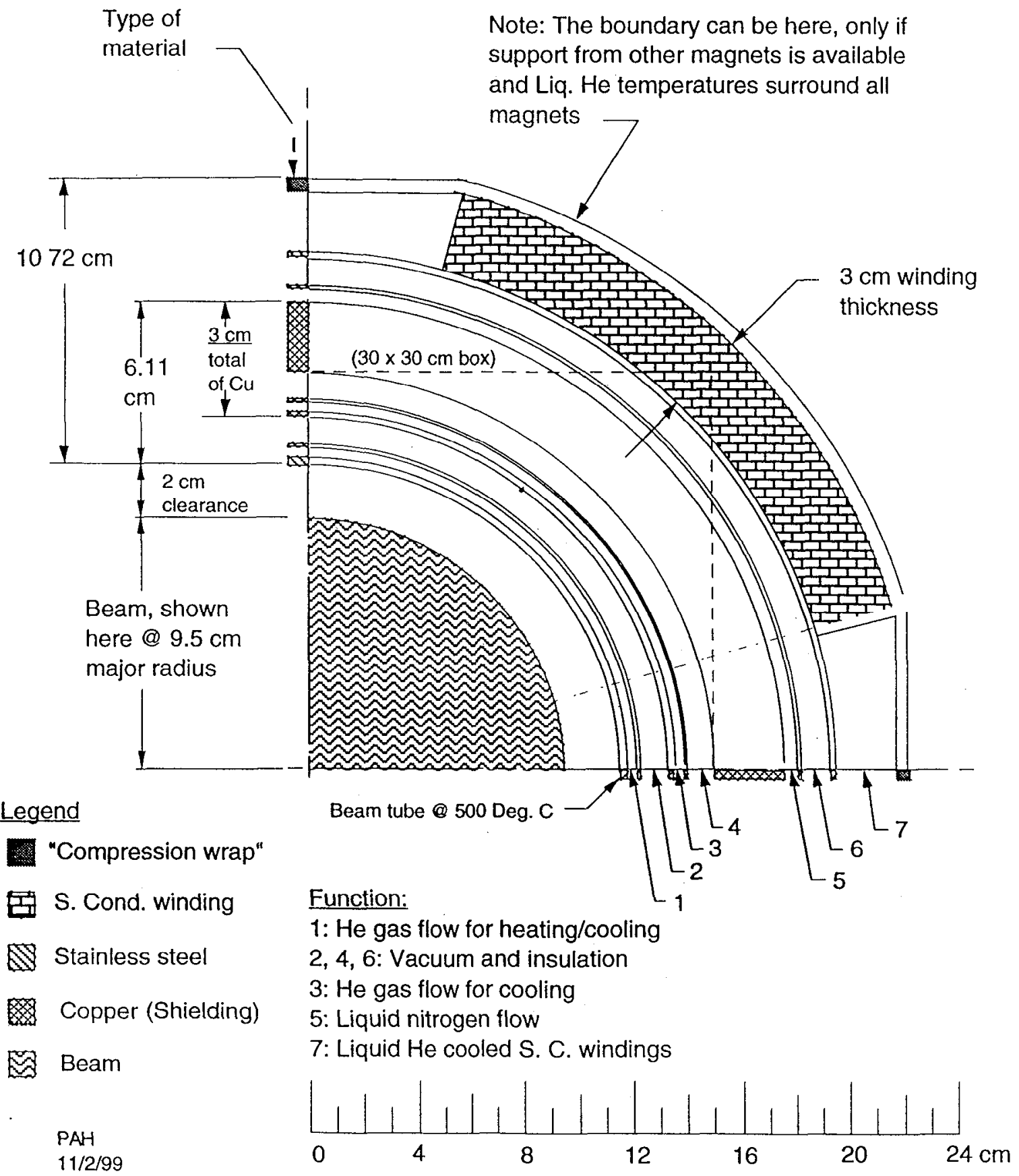

Figure 2. Magnet Q5 cross section 
View of inside surface of final focus magnets @ $5.18 \mathrm{~m}$ from shot point

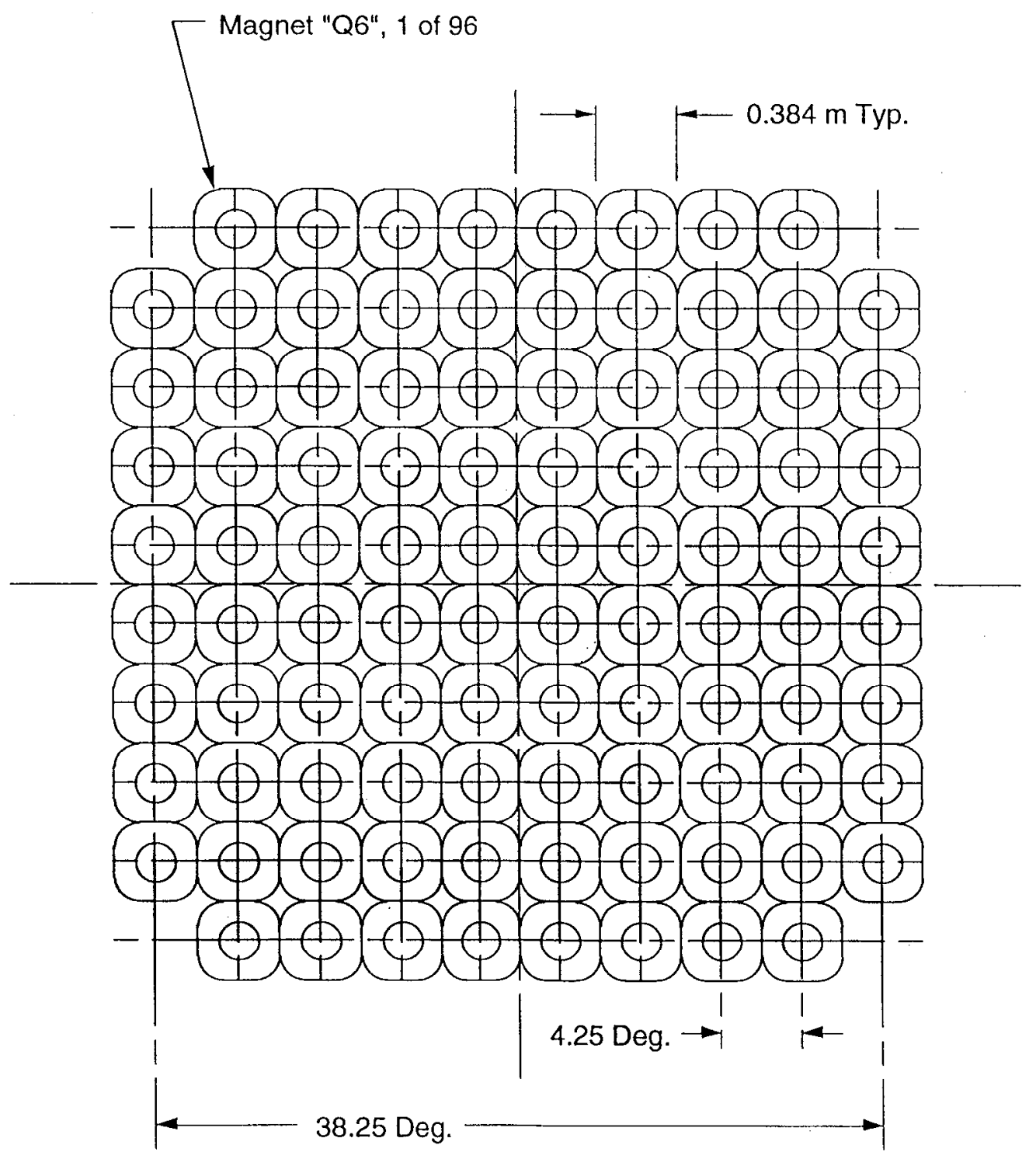

Figure 3. Q6 magnets at $5.18 \mathrm{~m}$ from the working point 


\section{Heavy lon Beam Lines With Magnets (cross section through the shot point)}

(This is the magnet configuration for horizontal and vertical shielding jets)

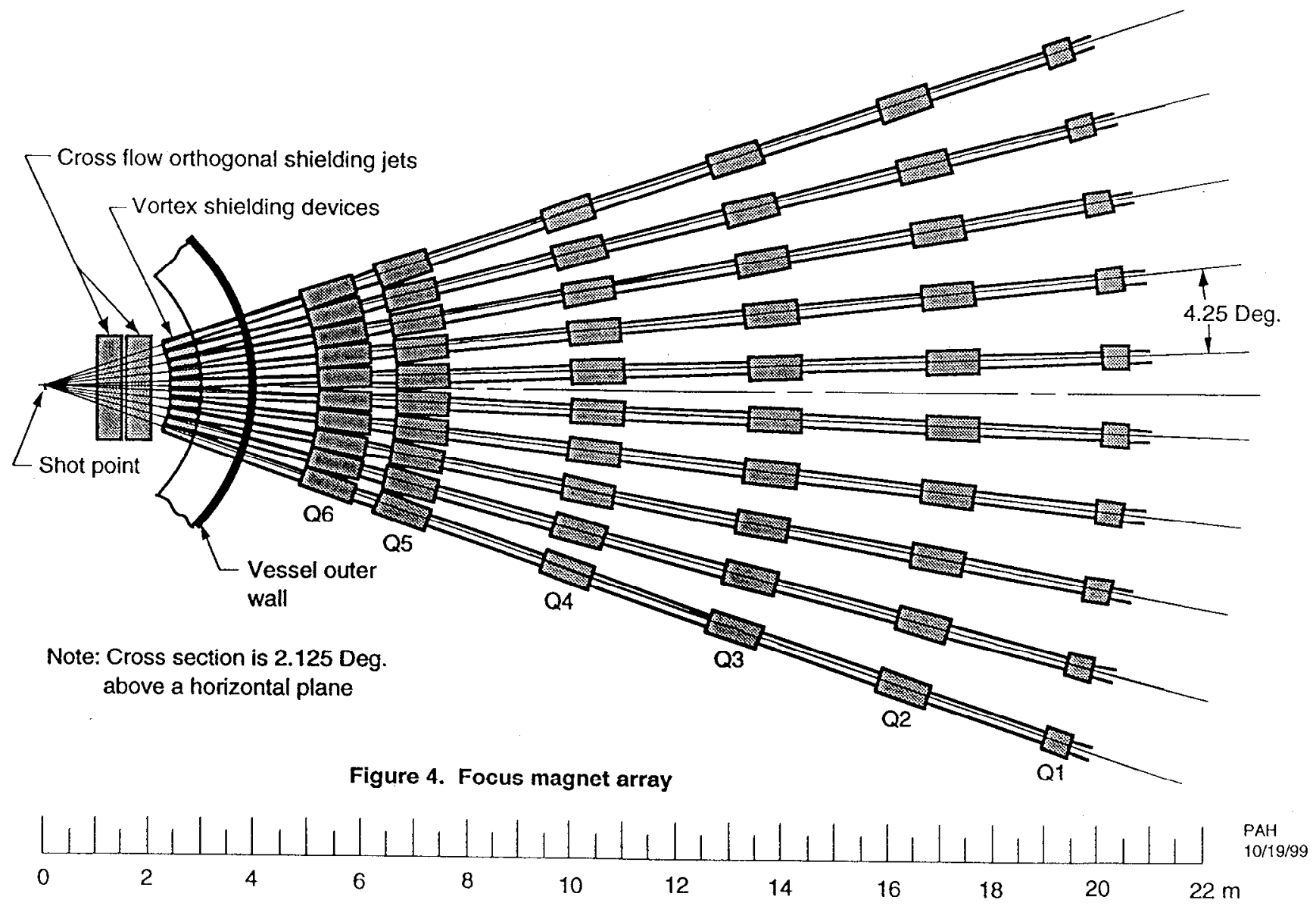


"Horizontal" jet at $1.6 \mathrm{~m}$ from W.P. $12 \mathrm{~m} / \mathrm{s}$

(Beams are not adjusted to compensate for jet curvature)
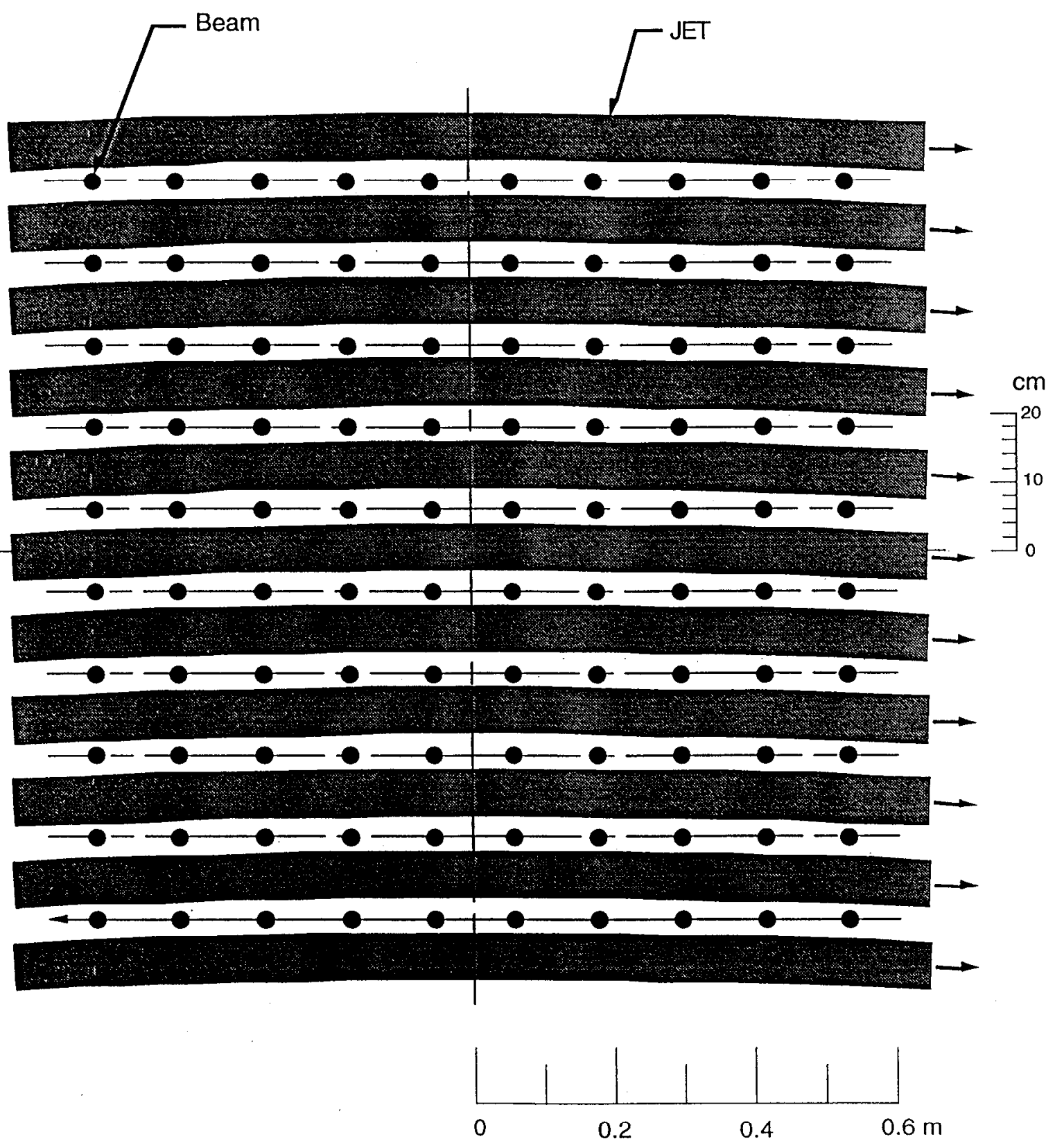

Figure 5. Horizontal shielding jets, beams evenly spaced 
"Horizontal" jets at $1.6 \mathrm{~m}$ from W.P., $12 \mathrm{~m} / \mathrm{s}$

(Beams are adjusted to compensate for jet curvature)

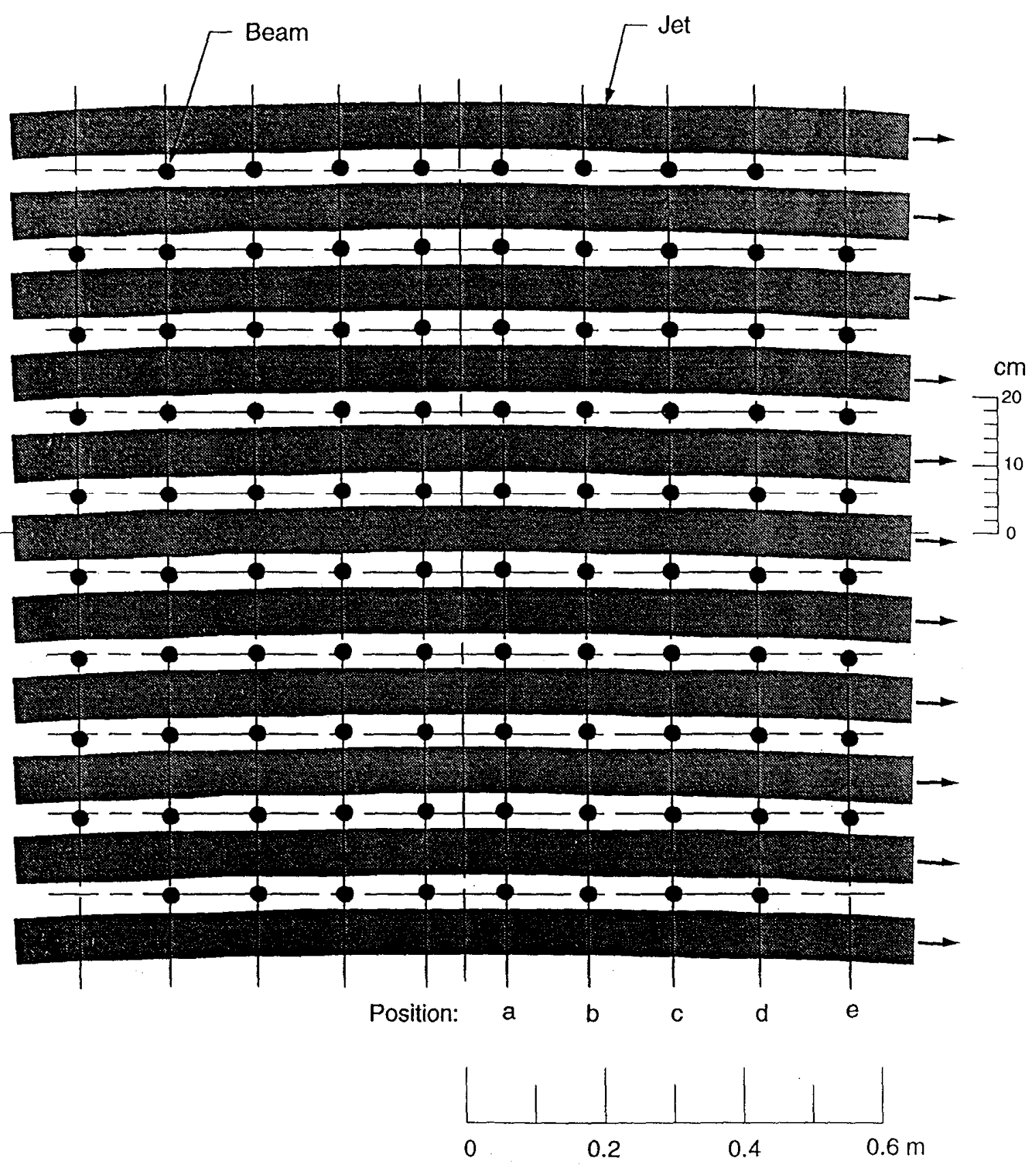

Figure 6. Horizontal shielding jets, beam positions adjusted for jet curvature 


\section{View of inside surface of final focus magnets @ $5.18 \mathrm{~m}$ from shot point}

(Magnets shifted for $12 \mathrm{~m} / \mathrm{s}$ "horizontal" jet curvature at $1.6 \mathrm{~m}$ from shot point)

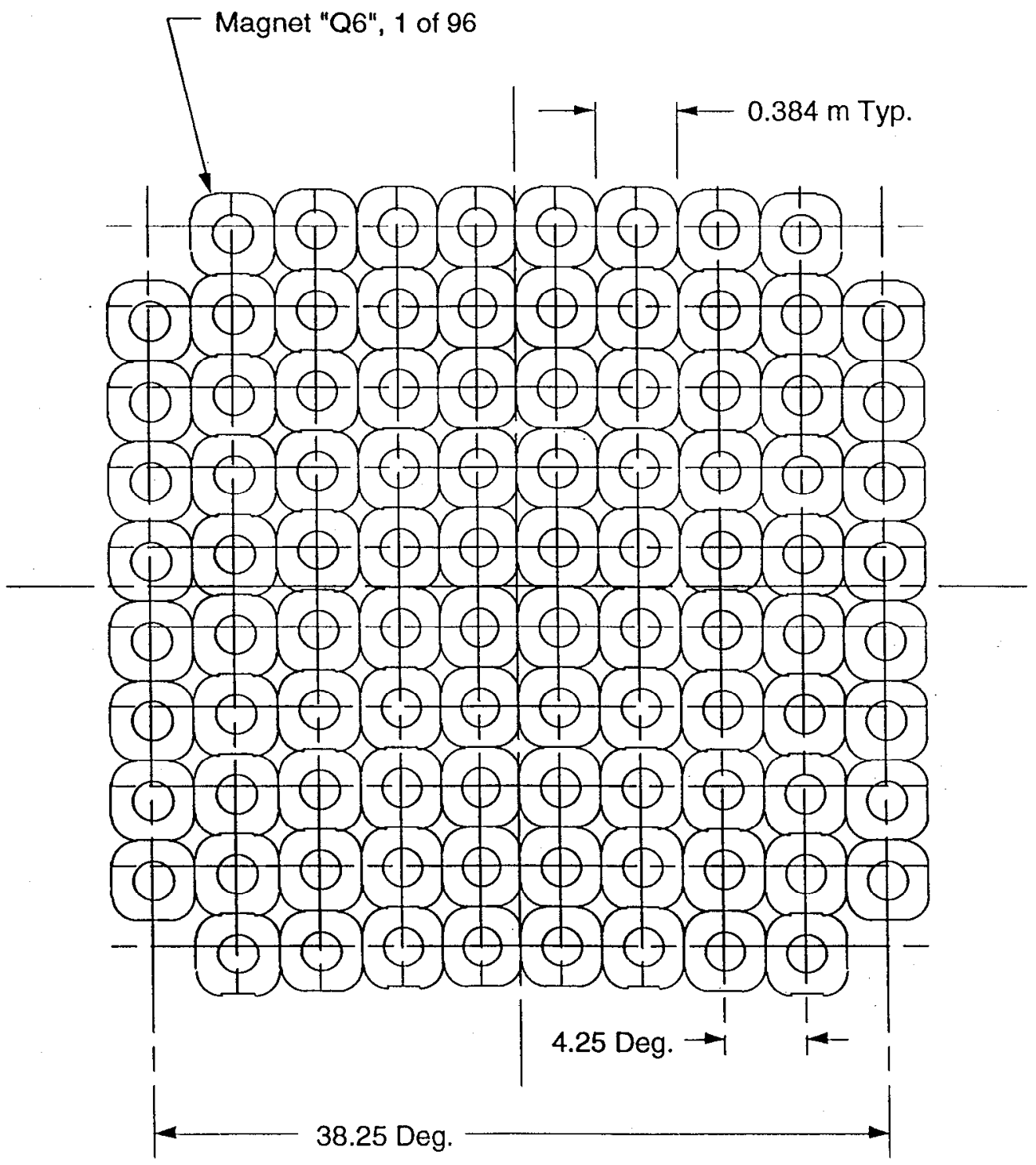

Figure 7. Q6 magnets (focus magnets shifted to compensate for jet curvature) 
Curvature of cross flow shielding jets from gravity (and spreading of jets: not shown)

causes shielding problems for the first wall and beamline shielding devices

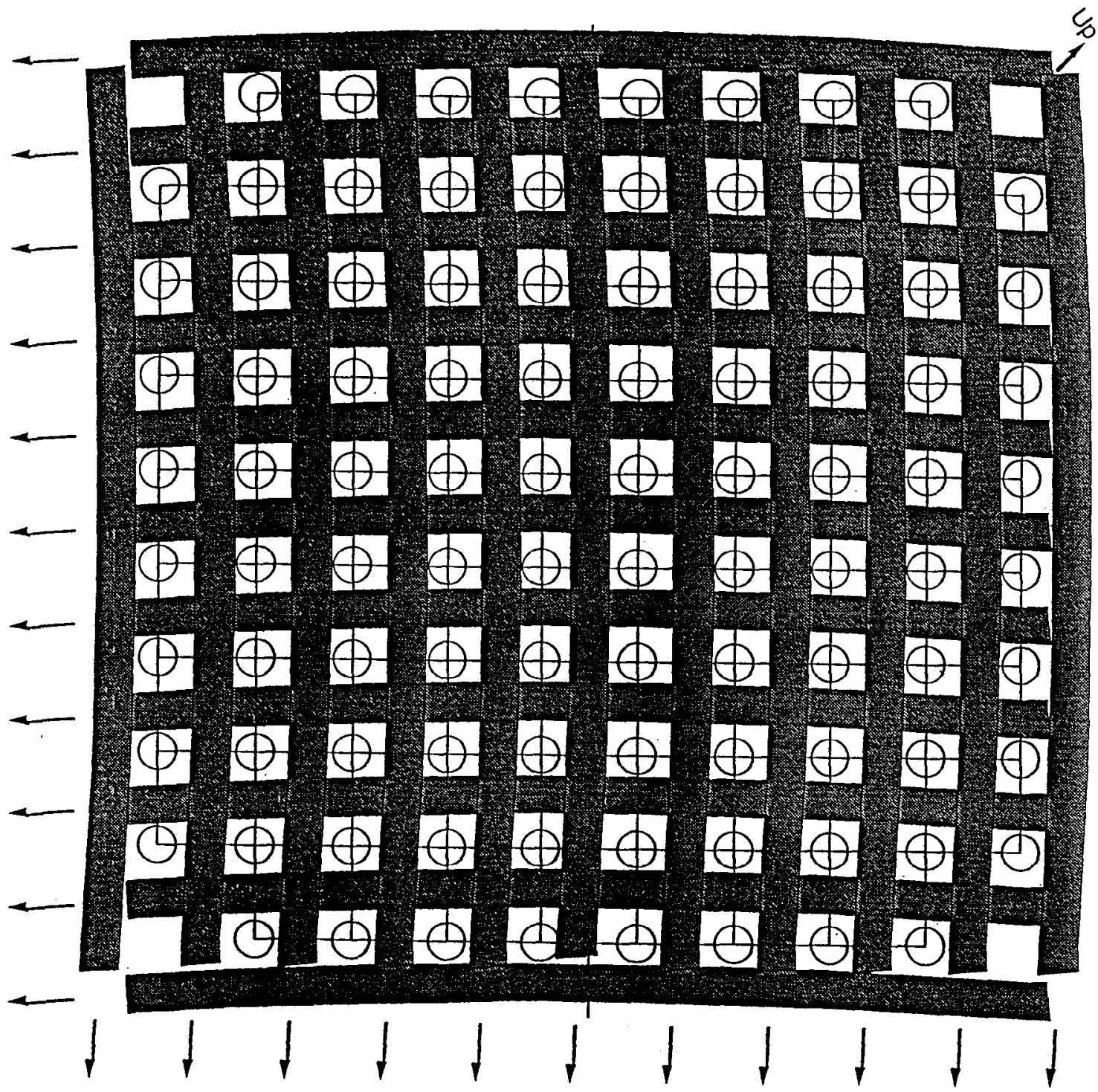

Note:

It may be practical to shift the beam trajectories, in order to center them between the jets

Figure 8. Cross jets at 45 Deg. from horizontal 